(Aus dem physiologischen Institut der Universität Kiel.)

\title{
Über scheinbare Atmung abgetöteter Zellen durch Farbstoffreduktion.
}

\author{
(Versuche an Acetonhefe.) \\ Von
}

Otto Meyerhor.

I.

Seit den bekannten Versuchen Ehrlich's ${ }^{1}$ ) ist die Farbstoffreduktion der versehiedensten Zellarten von zahlreichen Forschern untersucht und eingehend beschrieben worden. In diesen Forschungen sind zwei Richtungen zu unterscheiden, die zwar nicht in ausgesprochenem Gegensatz stehen, deren Versuchsergebnisse und Deutungen sich aber nicht ganz leicht miteinander vereinigen lassen. Ehrlich sieht in dem zutage tretenden Reduktionsvermögen der lebenden Zellen gegen Vitalfarbstoffe einen Ausdruck ihres dem normalen Stoffwechselvorgang entsprechenden Sauerstoffbedürfnisses, woraus er die Topographie und Intensität der vitalen Verbrennungsprozesse abliest, ähnlich deutet gleichzeitig Dreser ${ }^{2}$ ) die Methylenblaureduktion im Nierengewebe als einen "Heisshunger der tätigen Drüsenzellen nach Sauerstoff", - während Untersuchungen besonders auf bakteriologischer Seite damit zunächst schwer zu vereinigende Resultate geliefert haben. So fanden z. B. Wolff ${ }^{3}$ ) und andere, dass anaerobe Bakterien ebenso gut oder besser die verschiedenen Farbstoffe, insbesondere auch Methylenblau reduzieren als aerobe [nach K lett ${ }^{4}$ ) auch tellurige und selenige Salze zu Metallen], Catheart ${ }^{5}$ ) und

1) Das Sanerstoffbedürfnis des Organismus. Berlin 1885 .

2) Zeitschr. f. Biol. Bd. 3 S. 57 ff. 1885.

3) Zentralbl. f. Bakteriol. Bd. 27 S. 849 . 1900

4) Zeitschr. f. Hygiene Bd. 33 S. 137.

5) Arch. f. Hygiene Bd. 44 S. 295. 1902, und Münchener med. Wochenschr. Bd. 49 S. 595. 1902. Allerdings ist daran zu denken, dass nach $P$ feffer, Pflanzenphysiologie Bd. 1 S. 549, anaerobe Bakterien ebenfalls vorhandenen Sanerstoff aufzehren. 
Über scheinbare Atmung abgetöteter Zellen durch Farbstoffreduktion. 251

Hahn konnten dasselbe für fakultativ anaerobe bestätigen, wo die anaerob gezüchteten Bakterien ein grösseres Reduktionsvermögen für Methylenblau besassen als die aeroben Bakterien derselben Spezies. Ferner aber trat gegen die "Vitalität" des Reduktionsvermögens für Methylenblau, das schon zum Unterscheidungsmerkmal lebender von toten Zellen vorgeschlagen wurie ${ }^{1}$ ), die Beobachtung von $\mathrm{Hahn}$ auf, dass nach dem Acetonverfahren getötete Hefe, ebenso behandelte und nachier im Vakuum erhitzte sterile Bakterien, ja auch Hefepresssaft, ein sehr starkes Reduktionsvermögen für Methylenblau zeigen, das alle Eigenschaften mit dem der lebenden Zellen gemeinsam hat, Vernichtung durch Hitze, durch Gifte, Verhalten bei Zusätzen, Verdünnungen, Altern usw. ${ }^{2}$ ).

Auf das Reduktionsvermögen gegen Methylenblau wollen wir uns im nachfolgenden beschränken. Auf so verschiedenartigen Ursachen, wie ausgeschiedenen Stoffwechselprodukten, rein chemischen Umsetzungen usw., auch manche Reduktion von Metallsalzen oder einzelnen Farbstoffen beruhen mag, für die Methylenblaureduktion ist es durch $\mathrm{Hahn}$ sicher gestellt, dass sie durch die Leibessubstanz der Zellen geschieht, und ferner, dass sie durch ihre Ubiquität und andere Ejgenschaften am allermeisten von allen Farbredultionen Ähnlichkeiten mit dem normalen Atmungsvorgang verrät.

Nachdem nun kürzlich gezeigt war ${ }^{2}$ ), dass mit Aceton getöteten Bakterien und Seeigeleiern tatsächlich noch eine gut messbare Sauerstoffatmung zukommt, schien ein weiterer aus den Versuchen Hahn's gezogener Einwand gegen die Gleichsetzung der Methylenblaureduktion mit dem Sauerstoffverbrauch im tierischen Verbiennungsprozess zu entfallen. Endlich aber musste die grosse Zahl von Anhäugern einer derartigen Auffassung und die in diesem Sinn gefertigten Untersuchungen ${ }^{4}$ ) die Aufmerksamkeit darauf lenken, ob hier die Forschung

1) Neisser und Wechsberg, Münchener med. Wochenschr. $1900 \mathrm{Nr} .37$. Dass die Methode zur praktischeñ Erkennung lebender Bakterien oder Leukocyten geeignet sein kann, soll den Autoren natürlich nicht bestritten werden.

2) A. a. O. und die Zymasegärung von E. Buchner, H. Buchner und M. Hahn S. 341. 1903.

3) Warburg und Meyerhof, Pflüger's Arch. Bd. 148 S. 295. Das Optimum der Reduktion der Acetonbakterien ist jedoch nach $\mathrm{Hahn}$ bei $40^{\circ}$, das der Atmung etwa bei $30^{\circ}$.

4) Vgl. z. B. Unna; Arch. f. mikrosk. Anat. Bd. 78 S. 1. 1911, und die dort angeführte Literatur. 
einen Weg einschlug, der zur Aufklärung der vitalen Oxydationsvorgänge führen könnte. Aus all diesen Gründen schien eine kritische und experimentelle Studie angebracht, ob die Gleichsetzung von Farbstoffreduktion und Atmung berechtigt sei. Die folgende Arbeit euthält nun wenigstens für ein Objekt, die Acetonbefe, den Beweis, dass die durch Metbylenblau bewirkte Oxydation in der Zelle nach der quantitativen Seite etwas yon dem Atmungsvorgang Unabhängiges ist.

Die Versuche wurden im Zusammenhang mit den von 0 . Warburg und mir kürzlich veröffentlichten Untersuchungen über die Atmung abgetöteter Zellen und Zellfragmenten ${ }^{1}$ ) in der medizinischen Klinik zu Heidelberg begonnen und dann in Kiel fortgesetzt. Es war zugleich auch die Absicht, auf eine unabhängige Sauerstoffatmung der Acetonhefe zu fahnden; nacbdem aber diese an anderen in Aceton getöteten Zellen festgestellt war und sich auch zwei Arbeiten aus dem Pallad in'schen Institute fanden ${ }^{2}$ ), in denen bereits selbständiger Sauerstoffverbrauch von Acetonhefe gemessen war, trat diese Aufgabe mehr in den Hintergrund. Es fand sich auch bei mir in neutraler und schwach alkalischer Lösung stets eine kontinuierliche selbständige Sauerstoffzehrung bei - auf ihre Sterilität geprüfter - Acetonhefe, die bei den versehiedenen Präparaten wechselnd, bei den einzelnen aber ziemlich gleich war, so dass sich die Angaben der russischen Autoren bezüglich der Sauerstoffatmung von Acetonhefe bestätigen liessen. Diese Atmung beträgt im günstigsten Fall bei frisch hergestelltem Präparat etwa 2,0 bis 2,5\% der Atmung intakter Presshefe, meist nur etwa halb so viel. Da aber nach den Buchner'schen Zahlen die Gärung der Acetonhefe auch nur $1-2 \%$ der Ausgangshefegärung entspricht ${ }^{3}$ ), so ergibt sich das bemerkenswerte Resultat, dass der Abfall der Sauerstoffatmung dureb dieAcetonbehandlung nicht stärker ist als der der Gärung.

Die Versuche mit Methylenblau nahmen von folgender Beobachtung Hahn's ${ }^{4}$ ) ihren Ausgangspunkt. Setzt man zu einer

1) A. a. 0 .

2) Telesnin, Zentralbl. f. Bakteriol. II Bd. 12 s. 205.1904. Warschawsky, Zentralbl. f. Bakteriol. II Bd. 12 S. 400.

3) $1 \mathrm{~g}$ Acetonhefe entsteht aus $3 \mathrm{~g}$ Ausgangshefe.

4) Zymasegärung S. 344 . 
bestimmten Menge Acetonhefe in Wasser oder Bouillon eine abgemessene Menge Metbylenblaulösung, so wird diese in einer bestimmten Zeit entfärbt; fügt man jetzt dieselbe Methylenblaumenge nochmals zu, so wird auch diese und zwar in der gleichen Zeit. entfärbt wie die erste usw. (bei Presssaft nehmen die Redulktionszeiten bei weiteren Zusätzen stets zu). Dadureh wird der Gedanke nabe gelegt, die kontinuierliche und gleichmässige Reduktion durch Acetonhefe sei durch nichts anderes als den gefundenen Sanerstoffbedarf der getöteten Hefezellen bedingt, der ja, wie erwähnt, zum Teil trotz der Abtötung fortdauert. Der Versuch führte jedoch zu einem ganz anderen Ergebnis: Versorgt man die mit Methylenblau versetzte Acetonhefe hinlänglich mit Sauerstoff, soverbraucht siewährend etwa $2 \mathrm{Stundendas} \mathrm{doppelte}$ bis dreifacbe an Luftsauerstoffals ohne Methylenblau. Es finden also bei Methylenblaugegenwart Oxydationen statt, die ohne dasselbe nicht statthaben. Demgemäss lässt sich ebenfalls zeigen, d a s s im gegen Luft abgeschlossenen Raume die Reduktion. des Methylenblaus durch Acetonhefe schonzu einer Zeit stattfindet, wo die Acetonhefe in farbfreier Lösungnoch nicht allen Sauerstoff aufgezehrt hätte. Da die. gebildete Leukobase bei Sauerstoffgegenwart nicht beständig ist, beweist die einsetzende sichtbare Reduktion die Abwesenheit von Sauerstoff. Diese Abwesenheit entspringt jedoch nicht allein aus dem selbständigen Sauerstoff bedürfnis der Acetonhefe, sondern vielmehr aus der durch die Farbstoffgegenwart veranlassten Oxydation.

Dahin gehört ferner, dass gleiche Gewichtsteile lebender Hefe in Bouillon langsamer Methylenblau reduzieren als Acetonhefe, während sie durchsebnittlich $30 \mathrm{mal}$ so stark atmen. Während bei Acetonhefe die Reduktion beginnt, ehe der für gewöhnlich verbrauchte Sauerstoff aufgezehrt ist, wird dieselbe umgekehrt bei lebender Hefe erst sichtbar, nachdem sch on lange aller Sauerstoff aus der Lösung verschwunden sein muss, und setzt also keiveswegs die stockende Oxydation unmittelbar fort. Entsprechend diesem. kolossalen Übergewicht der Atmung der intakten Hefe über dieReduktion ist ein plus an Sauerstoffverbrauch der lebenden Hefe durch Methylenblaugegenwart nicht festzustellen.

Die Sauerstoffmessungen liessen sich durch Wärmemessungen ergänzen, und hier ergab sich das weitere Resultat, dass unter den für den Sauerstoffversuch geltenden Bedingungen, d. h. bei neutraler 
und schwach alkalischer Reaktion, eine dem selbständigen Sauerstoffverbrauch entsprechende Wärmebildung nachweisbar ist, dass diese aber bei Methylenblauzusatz gesteigert werden kann. Zum Unterschied liess die Wärmemessung in saurer Lösung (Acetonhefe, die stets sauer reagiert, in Wasser oder Bouillon) überhaupt keine oder nur eine ganz geringe selbständige "Atmung“" erkennen, während bei Methylenblauzusatz eine gut messbare Wärmetönung auftrat. Bei einer allerdings nicht ganz unbeträchtlichen methodischen Fehlerbreite ergab sich für die farbfreie Lösung das Resultat, dass sowohl bei Sauerstoffanwesenheit wie -abwesenheit das Verhältnis der erzeugten Wärme zur gebildeten Kohlensäure gut dem berechneten "kalorischen Gärungsquotienten" $\frac{g \text { cal. }}{\mathrm{mg} \mathrm{CO}_{2}}$ entsprach. Dies war dann natürlich bei Sauerstoffversorgung unter Methyleublauzusatz nicht mehr der Fall.

$\mathrm{Ob}$ sich entsprechend dem durch Methylenblau hervorgerufenen Sauerstoffverbrauch eine Kohlensäureausscheidung nachweisen lässt, konnte an diesem Objekte infolge. der enormen Kohlensäurebildung durch Gärung nicht studiert werden. Dagegen ist eine beträchtliche Stimulierung der Kohlensäureausscheidung durch Methylenblauzusatz bis über $100 \%$ im Palladin'schen Institute bei lebenden Samen und Stengelspitzen festgestellt ${ }^{1}$ ), eine Beobachtung, die den hier mitgeteilten an die Seite gestellt werden kann. Die Charakteristika der normalen Atmung, der zeitlich kontinuierliche und gleichmässige Sauerstoffverbrauch, Wärmebildung - und unter Umständen Kohlensäureausscheidung - sind also durch Methylenblauzisatz hervorzurufen, und doch wird eine kritische Diskussion ergeben, dass nach den mitgeteilten Versuchen und anschliessenden Überlegungen diese "scheinbare Atmung" anders zu deuten ist.

Es lag nicht im Felde dieser Arbeit, die Gesetze der Reduktionsfăhigkeit gegenüber Methylenblau näher zu untersuchen. Dies ist von $\mathrm{Hahn}$ und $\mathrm{Catheart}$ für Acetonhefe und Bakterien in umfangreicher Weise geschehen. Nur einzelne Punkte seien noch erwähnt. So beschreiben Palladin und seine Mitarbeiter eine abschwächende Wirkung von $\mathrm{Na}_{2} \mathrm{HPO}_{4}$ auf die Kohlensäurestimulierung. Dies liess sich auch bei dem durch Methylenblau bewirkten Sauerstoffverbrauch

1) Palladin, Hübbenet und Korsakow, Biochem. Zeitschr. Bd. 35 S. 1. 1911. 
beobachten. - Durch $\mathrm{Hahn}$ ist es bekannt, dass Bouillon gegenuber Wasser und schwach alkalische Reaktion gegenüber saurer die Reduktion sehr beschleunigt ${ }^{1}$ ). Obwohl sich dies sehr genau bestätigen liess, so zeigte sich doch kein deutlicher Unterschied des Sauerstoffverbrauchs in Bouillon und Wasser (beide spurenweise alkalisch), so dass die Grösse des Sauerstoffverbrauchs nicht unter allen Umständen der Geschwindigkeit der Reduktion parallel zu gehen scheint. - Die Reduktionsfähigkeit der verschiedenen benutzten Acetonpräparate war ziemlich verschieden; zum Teil erklären sich aber Differenzen wohl auch aus den niemals ganz gleichen Konzentrationen der Hefe und den Versehiedenheiten der Reaktion. Die zugesetzte Menge Methylenblau blieb in gewissen Grenzen $(0,1-0,4 \mathrm{~cm} 1 \%$ M. auf $0,3 \mathrm{~g}$ Acetonhefe in $2-3 \mathrm{ccm}$ Flüssigkeit) ohne deutlichen Einfluss.

Mit Presssaft konnten in Heidelberg nur einige orientierende Versuche vorgenommen werden, die ebenfalls einen auf die Methylenblaugegenwart zu beziehenden Sauerstoffkonsum erkennen liessen. In Kiel war keine Gelegenheit zur Herstellung von Hefepresssaft vorhanden.

Indigotin wird durch Acetonbefe bei sehwach alkalischer Reaktion auch reduziert, jedoch langsamer als Methylenblau; dementsprechend fand sich dadurch auch eine geringe Vermehrung der Sauerstoffzehrung.

Endlich sei noch ein Faktum hervorgehoben, das wenigstens methodisch von Wichtigkeit ist. Während bei der benutzten spurenweise alkalischen Reaktion Methylenblau allein sowie Methylenblau in Bouillon keinerlei Sauerstoffverbrauch zeigen, nimmt Methylenblau allein in alkalischer Lösung (z. B. 1/20 n. NaOH) grössere Mengen Sauerstoff auf unter allmählicher Violettverfärbung. Es bildet sich hierbei, wie aus den Untersuchungen Bernthsen's ${ }^{2}$ ) über die Methylenblaugruppe folgt, ein Gemisch von Metbylenviolett und Methylenazur. Während bei ersterem eine Dimethylamidogruppe unter Dimethylaminbildung durch ein O-Atom ersetzt wird, ist letzteres ein Oxydationșprodukt des Methylenblans, in dem der

1) Siehe besonders Arch. f. Hygiene Bd. 44 S. $307 \mathrm{ff}$.

2) Liebig's Annalen Bd. 230 S. 137. 1885, und Bd. 251 S. 1. 1889. Methylenblau (als salzsaures Salz) hat nach Bernthsen folgende Formel:

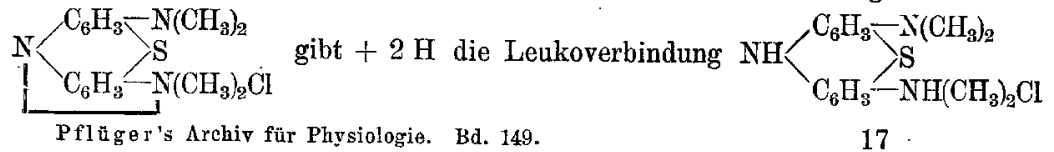


Schwefel 20 -Atome anlagert. Diese durch Analyse gefundene Tatsache wird nun durch den Sauerstoffverbrauch des alkalisch gemachten Methylenblaus sehr schön bestätigt. Der Sauerstoffverbrauch ist in alkalischer Bouillon noch erheblich grösser als in Wasser, was wohl als eine hinzutretende Reduktionswirkung der alkalischen Bouillon zu der Oxydation des Methylenblaus aufgefasst werden muss. (Eine "Autoxydation" unter eigener teilweiser Reduktion zur Leukobase nimmt Bernthsen auch für die Methylenazurbildung an. Dann dient der Sauerstoff der Luft dazu, die Leukobase wieder unter Wasserbildung in Methylenblau überzuführen.)

\section{Versuche.}

Sämtliche Versuche fanden in einem Wasserthermostaten von $29^{\circ}$ statt. Bei $40^{\circ}$ wäre der Unterschied zwischen Oxydation durch Methylenblau und selbständigem Sauerstoffverbrauch sicher noch grösser gewesen, da erstere dadurch vermehrt, letzterer dagegen auf die Dauer sehr herabgesetzt wird ${ }^{1}$ ), doch sollten möglichst. normale Bedingungen gewählt werden.

Die Acetonhefe wurde von A. Schroder, München, bezogen. Zwei Präparate, die für die meisten mitgeteilten Versuche benutzt wurden, ein frisch bezogenes und nicht behandeltes und ein älteres, das inzwischen kurze Zeit auf $100^{\circ}$ erhitzt war, wurden auf ihre Sterilität geprüft und erwiesen sich als völlig steril ${ }^{2}$ ). Von den übrigen war dies auch anzunehmen, ausser einem mit Stäbchen verunreinigten, das nicht weiter benutzt wurde. (Ein anderes war in Spuren mit. Sarcine verunreinigt, die aber jedenfalls mit fixiert waren.) Als Hefevergleich wurde obergärige Presshefe aus der Hefefabrik von Leptien, Kiel, benutzt, die ähnlich abgepresst war wie die Ausgangshefe des Acetonpulvers, bei dem $80 \mathrm{~g} 250 \mathrm{~g}$ Ausgangshefe entsprachen. Dies empfahl sich mehr als untergärige Bierhefe zu nehmen, weil keine Presse zur Verfügung stand.

Für die Sauerstoffmessungen wurde das kürzlich beschriebene Verfahren benutzt ${ }^{3}$ ). Als Atmungsgläser, in denen zugleich die

1) Vgl. Warburg u. Meyerhof, a. a. O.

2). Herrn Dr. Wagner im Hygien. Institut Kiel, der diese Prüfung vornahm, danke ich auch hier bestens dafür. Es wurden mehrere Ösen Acetonhefe in absteigenden Verdünnungen in je drei Bierwürze-Gelatine-Röhrchen verrieben und auf Platten ausgegossen. Nach 3 Tagen war bei $22^{\circ} \mathrm{C}$. nichts gewachsen.

3) Warlugrg und Meyerhof, a. a. 0. 
Messung stattfand, dienten Gefässe, in die, wie angegeben, ein Röhrchen eingeschmolzen war, das mit konzentrierter Natronlauge zur Absorption der Kohlensäure gefüllt wurde. Diese Gläser wurden in dem $\mathrm{Haldane-Barcroft-Manometer} \mathrm{befestigt,} \mathrm{an} \mathrm{dem,} \mathrm{wie.}$ häufig beschrieben ${ }^{\mathbf{1}}$ ), der Sauerstoffverbrauch durch Druckabnahme bestimmt wird.' Während des Versuchs mussten die Gläser dauernd in Bewegung gehalten werden, damit die am Boden befindliche Flüssigkeit mit Luft gesättigt bleibt. Es wurde deshalb teils mit der Hand geschüttelt; teils automatisch durch anstossende, vom Rührer bewegte Metallstäbe, ein Verfahren, das ebenso wie die meist benutzten kegelförmigen Gefässe von Herrn Dr. Si ebeck, Heidelberg, erprobt war. Je nach der Art der benutzten Gefässe, die verschiedenes Volumen hatten, wurde $1 \mathrm{ccm} \mathrm{n.} \mathrm{NaOH}$ oder $0,5 \mathrm{ccm}$ 2,5 n. $\mathrm{NaOH}$ benutzt.

Dies Verfahren gelingt jedoch wegen der starken Kohlensäurebildung (durch Selbstgärung) nur, wenn zu Versuchsbeginn sich keine Kohlensäure im Gasraum befindet, weil sonst die Absorption derselben innerhalb der Versuchszeit grosse Fehler gibt. Um diese Bedingung sicher zu erfülen, ist es nötig die Aufschwemmung der Acetonhefe selbst, die für sich immer sauer gegen Neutralrot und meist auch gegen Lackmus reagiert, soweit alkalisch zu machen, dass sie spurenweise rosa gegen Phenolphthalein ist, weil dann sicher keine Kohlensäure aus der Lösung austritt. Dass sich dann während des Versuchs durch Kohlensäurebildung die Reaktion wieder verschiebt und auch eventuell Kohlensäure in den Gasraum tritt, schadet nichts mehr, weil diese Kohlensäure durch Bindung an die konzentrierte Natroulauge wieder verschwindet.

Es wurde zinkfreies Methylenblau nach Ehrlich von Grübled benutzt, meist in $1 \%$ iger Lösung.

\section{A. Sauerstoffiersuche.}

1. Vergleich des Sauerstoffverbrauchs mit und ohne Methylenblau.

In folgender Tabelle sind die zu verschiedenen Zeiten und mit verschiedenen Präparaten gewonnenen Resultate zusammengestellt. Jedesmal wird vorher die in Wasser oder Bouillon aufoeschwemmte

1) Siehe z. B. 0. WVarburg, Zeitschr. f. physiol. Chemie Bd. 69 S. 457. 1910. - O. Meyerhof, Pflüger's Arch. Bd. 146 S. 162.1912. 
Gesamtmenge der Acetonhefe durch Zusatz von $\frac{\mathrm{n}}{10} \mathrm{NaOH}$ in der angegebenen Weise neutralisiert, eingeteilt und je $2-3 \mathrm{ccm}$ davon benutzt. Versuchstemperatur $29^{\circ}$.

In der Tabelle sind angegeben: Dauer in Stunden, $m$ : Acetonhefe in Gramm, $F$ : Flüssigkeit in Kubikzentimetern, $W$ : Wasser, $B: 1 \%$ Fleichextraktbouillon (die Menge gilt abzüglich des zugesetzten Methylenblaus), dies für beide Parallelversuche geltend; ferner für die Versuche getrennt $M$ : zugesetztes $1 \%$ Methylenblau, $p$ : Druckabnahme in Millimetern Manometerflüssigkeit, $v$ : Volumen des Gasraums der Atmungsgefässe, cem $\mathrm{O}_{2}$ : verbrauchte Menge Sauerstoff in Kubikzentimetern von $0^{0}$ und $760 \mathrm{~mm}$, endlich $D$ : Überschuss des Sauerstoffverbrauchs mit Methylenblau über den olne Methylenblau in Kubikzentimetern $\mathrm{O}_{2}$.

Tabelle I. Acetonhefe.

\begin{tabular}{|c|c|c|c|c|c|c|c|c|c|c|c|}
\hline \multirow{2}{*}{ Nis. } & \multirow{2}{*}{ Dauer } & \multirow{2}{*}{$m$} & \multirow{2}{*}{$F$} & \multicolumn{3}{|c|}{$\begin{array}{l}\text { Ohne Methylen- } \\
\text { blau }\end{array}$} & \multicolumn{4}{|c|}{ Mit Methylenblau } & \multirow{2}{*}{ D } \\
\hline & & & & $p$ & $v$ & $\begin{array}{c}\mathrm{ccm} \\
\mathrm{O}_{2} \\
\end{array}$ & $M$ & $p$ & $v$ & $\begin{array}{c}\mathrm{ccm} \\
\mathrm{O}_{2}\end{array}$ & \\
\hline 1 & $2^{\text {h }} 00^{\prime}$ & $0,3 \mathrm{~g}$ & 1,5 cen $B$ & 27 & 38 & 0,093 & $0,4 \mathrm{cem}$ & 65 & 37 & 0,218 & 0,125 \\
\hline 2 & 1 h $02^{\prime}$ & $0,3 \mathrm{~g}$ & 2 com $W$ & 38 & 38 & 0,130 & $0,3 \mathrm{cenl}$ & 68 & 38 & 0,234 & 0,104 \\
\hline 3 & 2 h $20^{\prime}$ & $0,15 \mathrm{~g}$ & $2 \mathrm{con} \mathrm{B}$ & 56 & 12,5 & 0,063 & 0,2 cent & 148 & 12,5 & 0,168 & 0,105 \\
\hline $\left.4^{1}\right)$ & Oh $40^{\circ}$ & $0,12 \mathrm{~g}$ & 1,5 com B & 32 & 12,5 & 0,036 & $0,15 \mathrm{~cm}$ & 84 & 12,0 & 0,091 & 0,055 \\
\hline 5 & 0 h $40^{\prime}$ & $0,14 \mathrm{~g}$ & $2 \mathrm{cem} W$ & 20 & 12,5 & 0,023 & $0,15 \mathrm{cem}$ & 50 & 12,5 & 0,057 & 0,034 \\
\hline 6 & $1^{\text {h }} \quad 00^{\prime}$ & $0,3 \mathrm{~g}$ & $2 \mathrm{cem} \pi$ & 38 & 12,2 & 0,042 & 0,3 cent & 80 & 12,2 & 0,088 & 0,046 \\
\hline 7 & $1^{\text {h }} 30^{\prime}$ & $0,3 \mathrm{~g}$ & $2 \mathrm{ccm} W$ & 83 & 13,2 & 0,099 & $0,2 \mathrm{ccml}$ & 146 & 11,8 & 0,156 & 0,057 \\
\hline 8 & $1^{\text {h }} 40^{\prime}$ & $0,3 \mathrm{~g}$ & 2 coll B & 57 & 13,2 & 0,068 & $0,2 \mathrm{cell}$ & 115 & 11,8 & 0,123 & 0,055 \\
\hline
\end{tabular}

1) A cetonhefe vor 3 Tagen hergestellt. 
Über scheinbare Atmung abgetöteter Zellen durch Farbstoffreduktion. 259

2. Vergleich des Sauerstoffverbrauchs bei intakter Hefe.

Wegen der starken Atmung von Hefe wird entsprechend weniger Menge genommen. Es wird $0,5 \mathrm{~g}$ für Versuch $1,1,0 \mathrm{~g}$ für 2 in $100 \mathrm{ccm}$ Wasser aufgeschwemmt, durch $0,3 \mathrm{ccm} \frac{\mathrm{n}}{10} \mathrm{NaOH}$ spurenweise rosa gemacht gegen $\mathrm{Ph}$, je $2 \mathrm{ccm}$ benutzt und wie oben verfahren. Es ergab sich :

\begin{tabular}{|c|c|c|c|c|c|c|c|c|}
\hline \multirow{2}{*}{$\mathrm{Nr}$. } & \multirow{2}{*}{ Zeit } & \multicolumn{3}{|c|}{ Ohne Methylenblau } & \multicolumn{4}{|c|}{ Mit Methylenblau } \\
\hline & & $p$ & $v$ & $\operatorname{ccm~} \mathrm{O}_{2}$ & $M$ & $p$ & $v$ & $\operatorname{ccm~} \mathrm{O}_{2}$ \\
\hline 1. & 0 h $40^{\prime}$ & 34 & 18,0 & 0,040 & $0,2 \mathrm{ccm}$ & 29 & 13,0 & 0,034 \\
\hline 2. & $0^{\text {b }} 40^{\prime}$ & 75 & 12,7 & 0,086 & $0,3 \mathrm{ccm}$ & 68 & 12,7 & 0,078 \\
\hline
\end{tabular}

3. Reduktionsvergleich.

Ein gut reduzierendes Präparat, $1 \mathrm{~g}$ Acetonhefe auf $10 \mathrm{ccm}$ Bouillon neutral, reduzierte $0,2 \mathrm{~cm} 1 \%$ iges Methylenblau in 15 Minuten zur totalen Entfärbung, die gleiche Menge Acetonhefe in $10 \mathrm{ccm}$ Wasser, $0,2 \mathrm{ccm} 1 \%$ iges Methylenblau in 30 Minuten. Ein schlechter reduzierendes Präparat 0,5 g Acetonhefe in $5 \mathrm{ccm}$ Bouillon neutral gegen Lackmus, reduzierte 0,5 cem $0,2 \%$ iges Methylenblau in 25 Minuten bis zur totalen Entfärbung. $0,5 \mathrm{~g}$ intakter Hefe in $5 \mathrm{ccm}$ Bouillon reduzierten die gleiche Menge Methylenblau in 30 Minuten. In Wasser dagegen brauchte $0,5 \mathrm{~g}$ dieser Acetonhefe über 2 Stunden; $0,5 \mathrm{~g}$ lebender Hefe ungefähr 2 Stunden.

Aus Sauerstofftitrationen nach Winkler und ebenso aus den Messungen nach obigem Verfahren ergibt sich, dass $1 \mathrm{~g}$ Hefe in einer Stunde in destilliertem Wasser oder $0,9 \% \mathrm{NaCl} 6-7 \mathrm{ccm}$ Sauerstoff verbraucht, wobei aber zu bemerken ist, dass die Atmung dabei rasch abfällt, so dass sie in der zweiten Stunde nur etwa halb so gross wie in der ersten ist (wohl aus Nahrungsmangel). Mithin ist der in $5 \mathrm{ccm}$ gelöste Sauerstoff von $0,5 \mathrm{~g}$ Hefe schon in etwa 25 Sekunden aufgezehrt, während es 30 Minuten in Bouillon, 2 Stunden in Wasser zur Reduktion bedarf.

Umgekehrt verbraucht $1 \mathrm{~g}$ Acetonhefe allein höchstens (bei frisch hergestelltem Acetonpulver) 0,45 cem pro 1 Stunde; meist nur etwa halb so viel (dagegen tritt keine so deutliche Abschwächung 
in der Zeit zutage). Der in 5 cem Bouillon gelöste Sauerstoff wird von $0,5 \mathrm{~g}$ allerfrühestens in 6-7 Minuten erschöpft, wo schon eine deutliche Aufhellung des Methylenblaus bemerkbar ist. Das schlechter reduzierende Präparat gebrauchte durch Atmung nur $0,15 \mathrm{cem} \mathrm{O}_{2}$ auf $1 \mathrm{~g}$ pro 1 Stunde; $0,5 \mathrm{~g}$ zehrten also in 5 ccm Flüssigkeit den Sauerstoff in 20 Minuten auf. Zu dieser Zeit war aber das Methylenblau in Bouillon schon fast vollständig entfärbt. Man sieht also, dass in diesem Fall die Reduktion schon lange eingesetzt hat, ehe der normaliter verbrauchte Sauerstoff aufgezehrt war; es wird eben infolge der Reduktion mehr Sauerstoff verbraucht als sonst. Im Falle der intakten Hefe dagegen wird eine Reduktion erst sichtbar, nachdem schon lange aller Sauerstoff erschöpft war, der schon in 25 Sekunden verschwunden sein muss.

Für einen Vergleich des selbständigen Sauerstoffverbrauchs der Acetonhefe mit intakter Hefe ist zu berücksichtigen, dass $1 \mathrm{~g}$ Acetonhefe $3 \mathrm{~g}$ Ausgangshefe entspricht. Wenn man, was wohl erlaubt ist, die Atmung der benutzten obergärigen Presshefe der gleich abgepressten Ausgangshefe der Grössenordnung nach gleich setzt, so beträgt die Atmung der Acetonhefe nur höchstens 2,5\% der Ausgangshefe, und zwar bei 3 Tage vor dem Versuch hergestelltem Pulver, wäbrend die Atmung bei gelagertem Pulver noch erheblich geringer ist. Doch ist, wie erwähnt, die Abschwächung der Gärung durch die Acetonbehandlung nach $\mathrm{Buchner}$ mindestens ebenso gross.

Folgende Sauerstoffversuche seien noch angefübrt:

\section{Einfluss von $\mathrm{Na}_{2} \mathrm{HPO}_{4}$.}

Je $0,3 \mathrm{~g}$ Acetonhefe in $2 \mathrm{ccm}$ Wasser (Ph. Spur rosa).

Zeit: $1^{\mathrm{h}} 30^{\prime}$.

Ohne Methylenblau . . . . $p=83 \quad v=13,2 \quad 0,099 \mathrm{ccm} \mathrm{O}_{2}$ Mit Methylenblau (0,2 cem) . $p=146 \quad v=11,8 \quad \mathbf{0 , 1 5 6}$ " " Mit Methylenblau und $0,5 \mathrm{ccm}$

$\mathrm{Na}_{2} \mathrm{HPO}_{4}$. . . . . . $p=126 \quad v=12,0 \quad \mathbf{0 , 1 3 7} "$ "

Je $0,3 \mathrm{~g}$ Acetonhefe in 2 cem Bouillon.

Zeit: $1^{\text {h }} 40^{\prime}$.

Ohne Methylenblau . . . . $p=57 \quad v=13,2 \quad \mathbf{0 , 0 6 8} \mathrm{ccm} \mathrm{O}$ Mit Methylenblau (0,2 cem) - $p=115 \quad v=11,8 \quad \mathbf{0 , 1 2 3}$ " " Mit Methylenblau und $0,5 \mathrm{ccm}$

$\mathrm{Na}_{2} \mathrm{HPO}_{4}$. . . . . . $p=102 \quad v=12,3 \quad \mathbf{0 , 1 1 3}$ " " 
Über scheinbare Atmung abgetöteter Zellen durch Farbstoffreduktion. 261

5. Sauerstoffverbrauch unter Indigotinwirkung $(1 \%)$.

Zeit: $40^{\prime}$. Je $0,12 \mathrm{~g}$ Acetonhefe in $1,5 \mathrm{ccm}$ Wasser.

Ohne Zusatz . . . . . . . . $p=32 \quad v=12,5 \quad 0,036 \quad c \mathrm{~cm} \mathrm{O}_{2}$

Mit $1 \%$ Methylenblau $(0,15 \mathrm{ccm}) \quad p=84 \quad v=12,0 \quad \mathbf{0 , 0 9 1} " n$

Mit $1 \%$ Indigotin $(0,15 \mathrm{ccm}) \cdot p=58 \quad v=12,5 \quad \mathbf{0 , 0 6 6} \quad$ "

Zeit: $1^{\mathrm{h}}$. Je $0,3 \mathrm{~g}$ Acetonhefe in $2 \mathrm{ccm}$ Wasser.

Ohne Zusatz . . . . . . . $p=38 \quad v=12,2 \quad \mathbf{0 , 0 4 2} \mathrm{ccm} 0_{2}$

Mit $0,3 \mathrm{cem} 1 \%$ Methylenblau $p=80 \quad v=12,2 \quad \mathbf{0 , 0 8 8}$ n n

Mit $0,3 \mathrm{ccm} 1 \%$ Indigotin . . $p=54 \quad v=11,2 \quad \mathbf{0 , 0 5 5}, \ldots$

6. Sauerstoffverbrauch durch Methylenblau allein in alkalischer Lösung. (Metbylenazurbildung).

a) $2 \mathrm{cem} 0,001 \mathrm{n} . \mathrm{NaOH}+1 \mathrm{cem} 0,2 \%$ iges Methylenblau gibt in $80^{\prime}$. . . . . $p=0$

$2 \mathrm{ccm} 0,1 \mathrm{n} . \mathrm{NaOH}+1 \mathrm{~cm} 0,2 \%$ iges $\mathrm{Me}-$

thylenblau gibt in $80^{\circ}$. . . . . $p=33 \quad v=11,0$

$1 \mathrm{ccm}$ Bouillon (neutr.) $+1 \mathrm{~cm} 0,1 \mathrm{n} . \mathrm{NaOH}$

+1 cem 0,2\% iges Methylenblau gibt in $80^{\prime} \quad p=52 \quad v=12,0$

$1 \mathrm{ccm}$ Bouillon $+1 \mathrm{ccm} 0,1 \mathrm{n} . \mathrm{NaOH}$ ohne

Methylenblau gibt in $80^{\prime}$. . . . . $p=1 \quad v=13,0$

1 cem Bouillon $+1 \mathrm{cem} 0,001$ n. $\mathrm{NaOH}$

$+1 \mathrm{ccm} 0,2 \%$ iges Methylenblau gibt in $80^{\prime} \quad p=6 \quad v=11,7$

b) 2 cem 0,05 n. $\mathrm{NaOH}+0,3 \mathrm{cem} 1 \%$ iges

Methylenblau gibt in $100^{\prime}$. . . . . $p=43 \quad v=11,7$

2 cem 0,05 n. $\mathrm{NaOH}+0,3 \mathrm{ccm} 1 \%$ iges

Methylenblau + 2Tropfen Phenolphthalein

gibt in $100^{\prime}$. . . . . . . . . . $p=63 \quad v=12,4$

c) $2 \mathrm{ccm} 0,0003 \mathrm{n} . \mathrm{NaOH}+0,3 \mathrm{ccm} 1 \%$ iges

Methylenblau gibt in $1^{\mathrm{h}} \cdot .+. \cdot p=0$

d) $2,4 \mathrm{ccm} 0,05$ n. $\mathrm{NaOH}+0,2 \mathrm{cem} 1 \%$ iges

Methylenblau gibt in $2 \mathrm{~h}$. . . . . $p=61 \quad v=12,0$

\section{B. Wärmeversuche.}

7. Intakte Hefe.

Alle Wärmemessungen fanden nach der früher beschriebenen Methode $^{1}$ ) mit Dewar-Gefässen statt. Es wurden erst einige orientierende Messungen an intakter Hefe vorgenommen, um die

1) Biochem. Zeitschr. Bd. 35 S, 265. 1911. Bezüglich einzelner Modifikationen für die Temperatur von $29^{\circ} \mathrm{C}$. und Sauerstoffdurchleitung siehe Pflüg er's 
Atmungswärme derselben zu bestimmen. Die Gärungswärme der Hefe ist schon von $R u b n$ er gemessen worden ${ }^{1}$ ). Schwemmt man die Hefe in $0,9 \% \mathrm{NaCl}$ mit oder ohne Zusatz von Hühnereiweiss auf, so ist nach ganz kurzer Zeit jede Spur Wärmebildung erloschen; hält man das Gefäss sanerstofffrei, wozu völlig genügt, es bis zum Rande $\mathrm{zu}$ füllen und wie gewöhnlich verschlossen einzuhängen, da dann aller noch vorhandener Sauerstoff sofort von der atmenden Hefe aufgezehrt wird, so lässt sich auch an Hefe das kürzlich an Gänseerythrocyten und Vibrio Metschnikoff erhaltene Resultat bestätigen, dass für mehrere Stunden keine Spur von Wärmebildung nachweisbarist, also der Energieumsatzvöllig sistiert, daweder Sauerstoff noch der Zymase zugängliches, vergärbares Material vorhanden ist ${ }^{2}$ ). Die Wärmeproduktion setzt aber sofort nach dem Einleiten von Sauerstoff ein, und sobald die anfangs gelöste Kohlensäure vertrieben ist, lässt sich der nun folgende Temperaturanstieg direkt und vollständig auf die Atmung beziehen.

So wurde erhalten:

a) $1 \mathrm{~g}$ Hefe in $240 \mathrm{ccm} 0,9 \%$ iger $\mathrm{NaCl}$ : ohne Sauerstoff während 55 Min. $0,00^{\circ}$
mit
$40 \# 0,157^{\circ}$

b) $1 \frac{1 / 2}{\mathrm{~g}}$ Hefe in $0,5 \% \mathrm{NaCl}, 10 \%$ igem Hühnereiweiss:

ohne Sauerstoff $2^{1 / 2}$ Stunde $0,00^{0}$

mit $" 1$ Stunde 20 Min. $0,310^{\circ}$

c) $1 \mathrm{~g}$ Hefe in $3 \% \mathrm{Na}_{2} \mathrm{HPO}_{4} 0,5 \% \mathrm{NaCl}$

ohne Sauerstoff während 2 Stunden 50 Min. $0,00^{\circ}$

mit

$"$

55 Min. $0,220^{\circ}$

Arch. Bd. 146 S. 161. Bei grösseren Sauerstoffmengen wurde für die Menge des durchgeleiteten Sauerstoffes entsprecbend seiner Wärmekapazität, der Differenz der Thermostatentemperatur und der im Dewar-Gefäss und der daraus sich berechnenden Verdampfung bzw. Kondensation in dem für Thermostatentemperatur wasserdampfgesättigten Sauerstoff eine Korrektur berechnet.

1) Arch. f. Hygiene Bd. 49 S. 355. 1904. Vgl. dazu auch Arch. f. Hygiene Bd. 48 S. 260 . 1904. In diesen Versuchen bediente sich Rubner ebenfalls evalkuierter Gefässe, die als "Mikrokalorimeter" bezeichnet wurden und die nach seiner Beschreibung Dewar-Flaschen oder diesen sehr ähnlich sind. Es war dies wohl die erste Anwendung Dewar'scher Gefässe für physiologische Zwecke (im übrigen ist seine Methode von der hier benutaten verschieden).

2) Dagegen ist für die obligat aeroben Zellen, Vibrio Metschnikoff und Gänseblutkörperchen wahrscheinlich wohl die Abwesenheit von Sauerstoff allein für die Sistierung des Enęrgieumsatzes ausreichend. 
Die Genauigkeit für die Zeit des Sauerstoffabschlusses ist etwa derart, dass. sicherlich weniger als $1,5 \%$ des späteren Energieumsatzes statt hatten. Ferner fand sich hier dasselbe Nachlassen der Oxydationswärme in NaCl-Lösungen mit der Zeit wie bei den Sauerstoffmessungen. Ein Versuch zụ annähernden Bestimmung des kalorischen Quotienten der Sauerstoffatmung ergab ungefähr 4. Auf eine genauere Bestimmung wurde kein Wert gelegt.

8. Wärmeversuche an Acetonhefe in saurer Lösung.

Schwemmt man Acetonbefe in Wasser, in NaCl-Lösung oder Fleischextrakt-Bouillon auf, so zeigt dieselbe im Gegensatz zur unversehrten Hefe eine erhebliche "Selbstgärung". Es beruht diese nach Bachner auf der Vergärung des in der Hefe enthaltenen Glykogens ${ }^{1}$ ), das erst, weil es selbst nicht diffundieren kann, bei Schädigung der strukturellen Organisation (so auch bei absterbender Hefe) mit der Zymase in Berührung kommt. Die Selbstgärung lässt anfangs sehr rasch, später allmählich langsamer nach. Für die Feststellung einer etwaigen Oxydationswärme der Acetonhefe wurde so verfahren: es wird der Temperaturanstieg in der Zeit ohne Sauerstoffdurchleiten mit dem während des Durchleitens verglichen. Es ist dabei folgendes in Betracht zu ziehen: als Temperaturanstieg ohne Sauerstoff gilt wegen des Nachlassens der Selbstgärung das Mittel zwischen dem vorherigen und nachherigen Anstieg. Wegen des Wegführens der Kohlensäure beim Durchleiten, die im Gegensatz dazu bei Sauerstoffabschluss grösstenteils gelöst bleibt, ist zu bemerken, dass pro $1 \mathrm{mg} \mathrm{CO}_{2}$ bei gasförmiger Entwieklung $0,29 \mathrm{~g}$ Kalorien, bei Lösung der Kohlensäure in Wasser 0,415 g Kalorien auftreten ${ }^{2}$ ) durch Gärung. Ist also durch ein anfänglich rascheres Sauerstoffdurchleiten die überschüssige Kohlensäure entfernt, so muss nun der fortlaufende Temperaturanstieg sich zu dem bei Sauerstoffabschluss verhalten wie $0,29 \mathrm{zu} 0,415$. Diese Bedingung ist tatsächlich fast genau in saurer Lösung erfüllt, und wir schliessen daraus, dass sich hier nur eine sehr geringe Oxydationswärme bilden kann.

1) Zymasegärung S. 92.

2) Zahlen dafür berechnet nach Rubner. Arch. f. Hygiene Bd. 49 S. 397. Die Zahlen gelten für Rohrzucker. Bei Glykogen kommt noch die Wärmetönung der Hydrolyse hinzu. (Diese beträgt, nach Rubner's Zahlen für die Inversion des Rohrzuckers pro $1 \mathrm{mg}$ Gärungskohlensäure etwa $0,02 \mathrm{~g}$-Cal und dürfte für die Hydrolyse des Glykogens von gleicher Grössenordnung sein.) 
Eine gewisse Fehlerbreite ist dadurch gegeben, dass ja auch ohne Durchleiten ein gewisser Vorrat an gelöstem Sauerstoff vorhanden ist. Diesem Einwand wurde in ein em Fall dadureh begegnet, dass in der Zwischenzeit Wasserstoff durchgeleitet wurde, und da dann auch der Unterschied der Gärungswärmen in Wegfall kommt, weil in beiden Fällen die Koblensäure weggeführt wird, so steht das gewonnene Resultat sehr gut mit den übrigen im Einklang: in diesem Falle war nämlich der Temperaturanstieg in Wasserstoff genau so gross wie in Sauerstoff. Für die anderen Fälle ist zu bedenken, dass der gesamte in Wasser gelöste Sauerstoff der Luft etwa $0,02^{\circ}$ Temperaturanstieg durch Oxydationsvorgänge hervorrufen kann. Dieser immerhin nicht unbeträchtlichen Ungenauigkeit wurde in einem weiteren Falle noch dadurch beigekommen, dass nach gleichzeitigen Kohlensäuremessungen die erzeugte Menge Kohlensäure in die gebildete Wärme dividiert, mit grosser Annäherung den kalorischen Gärungsquotienten der Kohlensäure ergab. Die Kohlensäuremessung fand ebenfalls mit Barcroft-Manometern statt, ebenso wie es kürzlich A. Dorner ${ }^{1}$ ) beschrieben hat. Es werden die gewöhnlichen Absorptionsgläser benutzt, jedoch die Kohlensäure nicht absorbiert, sondern umgekehrt durch Schütteln aus der Flüssigkeit getrieben; und ihre Menge berechnet sich dann aus den positiven Drucken des Manometers. Bedingung ist, dass die Flüssigkeit sauer gegen Lakmus reagiert. Die Genauigkeit des Verfahrens wurde durch gleichzeitige Messungen nach dem ursprünglichen Barcroft'schen Verfahren mit Weinsäurezusatz und entsprechender Vorkontrolle geprüft und bestätigt ${ }^{2}$ ).

Wurde nun zu der Flüssigkeit im De war-Gefäss Methylenblau zugesetzt, so wurde jetzt meist ein deutliches Plus, in anderen Fällen ein $z u$ berechnender Überschuss von Wärmebildung bei Sauerstoffdurchleiten gegenüber Sauerstoffabschluss festgestellt. Ein absoluter Vergleich dieser Wärmemenge mit den früher beschriebenen Sauerstoffzebrungen lässt sich allerdings wegen der Verschiedenheit vieler wichtiger Umstände (Menge, Konzentration der Stoffe, Reaktion usw.) nicht durchführen.

1) Zeitschr. f. physiol. Chemie Bd. 81 S. 99.1912.

2) Etwaige Sauerstoffatmung, die negative Drucke bewirken würde, schadet nichts, weil ja entsprechend dem verbrauchten Sauerstoff Atmungskohlensäure auftritt, was sich, da es ja absolut nur von kleinem Betrag sein kann, innerbalb der Fehlergrenzen ganz auf hebt. 
a) $25 \mathrm{~g}$ Acetonhefe zu $220 \mathrm{ccm}$ dest. Wasser

(Wasserwert 260) zeigt in 20' ohne Sauerstoffdurchleiten . . . . . . . $0,169^{\circ} \mathrm{C}$.

$15^{\circ}$ später in $20^{\prime}$ mit Sauerstoffdurchleiten $0,109^{\circ} \mathrm{C}$. $5^{\prime}$ später in $20^{\prime}$ obne Sanerstoffdurchleiten $0,140^{\circ} \mathrm{C}$.

Proportion : $\frac{\mathrm{CO}_{2} \cdot \text { Wegführung }}{\mathrm{CO}_{2}-\text { Lösung }}=\frac{0,109}{0,155}=\frac{0,29}{0,415}\left(\right.$ statt $\left.\frac{0,29}{0,415}\right)$.

Wiederholung:

b) $25 \mathrm{~g}$ Acetonhefe zu $220 \mathrm{~cm}$ dest. Wasser

zeigt in $20^{\prime}$ ohne Sauerstoffdurchleiten $0,112^{\circ} \mathrm{C}$.

$15^{\prime}$ später in 20' mit Sauerstoffdurchleiten $0,059^{\circ} \mathrm{C}$. $20^{\prime}$ ohne Sauerstoffdurehleiten . . . . $0,084^{\circ} \mathrm{C}$.

$$
\text { Proportion: } \frac{0,059}{0,098}=\frac{0,25}{0,415}\left(\text { statt } \frac{0,29}{0,415}\right) \text {. }
$$

c) $20 \mathrm{~g}$ Acetonhefe in $240 \mathrm{ccm} 1 \%$ Fleisch-

extrakt $30^{\prime}$ mit Sauerstoffdurchleiten . $0,084^{\circ} \mathrm{C}$.

$20^{\prime}$ später $30^{\prime}$ mit Wasserstoffdurcbleiten . $0,075^{\circ} \mathrm{C}$.

$5^{\prime}$ später $30^{\prime}$ mit Sauerstoffdurchleiten . . $0,060^{\circ} \mathrm{C}$.

$$
\text { Proportion: } \left.\frac{\mathrm{O}_{2}}{\mathrm{H}_{2}}=\frac{72}{75} \text { (statt } 1\right) \text {. }
$$

d) $20 \mathrm{~g}$ Acetonhefe in $220 \mathrm{ccm} 1 \%$ Fleisch-

extrakt zeigt in $20^{\prime}$ ohne $\mathrm{O}_{2}$-Durchleiten $0,066^{\circ} \mathrm{C}$. (25' später) in $20^{\prime}$ mit $\mathrm{O}_{2^{\prime}}$-Durehleiten . . $0,042^{\circ} \mathrm{C}$.

in $15^{\prime} 0,045^{\circ} \mathrm{C}$. ohne $\mathrm{O}_{2}$-Durchleiten . $0,060^{\circ} \mathrm{C}$. in $20^{\prime}$.

Proportion: $\frac{\mathrm{CO}_{2} \text {-Wegführung }}{\mathrm{CO}_{2} \text {-Lösung }}=\frac{0,042}{0,063}=\frac{0,28}{0,415}\left(\right.$ statt $\left.\frac{0,29}{0,415}\right)$.

Zur gleichen Lösung 2 cem $1 \%$ iger

Methylenblau zugesetzt gibt ohne

$\mathrm{O}_{2}$ in $40^{\prime} 0,064^{\circ} \mathrm{C}=$. . . . . . $0,032^{\circ} \mathrm{C}$. in $20^{\prime}$.

mit $\mathrm{O}_{2}$-Durchleiten in $20^{\prime}$. . . . . $0,030^{\circ} \mathrm{C}$.

$7^{\prime}$ später in $2 \mathrm{~h} 0,177^{\circ} \mathrm{C}=$. . . . $0,0295^{\circ} \mathrm{C}$. in $20^{\prime}$.

Proportion : $\frac{\mathrm{CO}_{2} \text {-Wegführung }}{\mathrm{CO}_{2} \text {-Lösung }}=\frac{0,030}{0,031}=\frac{0,40}{0,415}\left(\right.$ statt $\left.\frac{0,29}{0,415}\right)$;

also ein geringes Plus durch $\mathrm{O}_{2}$-Einleiten bei

Methylenblaugegenwart.

e) $18, \breve{s} \mathrm{~g}$ Acetonhefe $+230 \mathrm{ccm} 1 \%$ igen

Fleischextrakt. $4^{\mathrm{h}}$ ohne $\mathrm{O}_{2}$, davon die letzten $35^{\prime}(\alpha) 0,037^{\circ} \mathrm{C}=$. . . $0,032^{\circ} \mathrm{C}$. in $30^{\prime}$. 
$\left(40^{\prime}\right.$ später) $(\beta) \quad 35^{\prime}$ mit $\mathrm{O}_{2}$-Einleiten $0,0325^{\circ} \mathrm{C} .=$. . . . . . . . . $0,028^{\circ} \mathrm{C}$. in $30^{\prime}$.

Dazu 2,5 ecm $1 \%$ iges Methylenblau $30^{\prime}$ mit $\mathrm{O}_{2}$-Einleiten. . . . . . . $0,050^{\circ} \mathrm{C}$. $80^{\circ}$ ohne $\mathrm{O}_{2}$-Einleiten $0,091=$. . . $\mathbf{0 , 0 3 4}^{\circ} \mathrm{C}$. in $30^{\prime}$.

Die der ersten Versuchshälfte entsprechende $\mathrm{CO}_{2}$-Bildung beträgt (Zeit von $\alpha$ und $\beta$ ) für $1^{\text {h }} 40^{\prime} p=80, v=39, m=2 \mathrm{cem}$ gleicher Lösung, $=0,293 \mathrm{cem} \mathrm{CO}_{2}$ : entspricht $68,5 \mathrm{mg} \mathrm{CO}$ in der Menge des Wärmeversuchs (Wasserwert des Wärmeversuchs 262). Die Ausrechnung ergibt für die Zeit des $\mathrm{O}_{2}$-Finleitens $\mathrm{Q} \frac{\mathrm{g} \text { cal }}{\mathrm{mg} \mathrm{CO}_{2}^{-}}=0,34$ (statt 0,29 ), des $\mathrm{O}_{2}$-Abschlusses $\mathrm{Q} \frac{\mathrm{g} \mathrm{cal}}{\mathrm{mgCO}}=0,40$ (statt 0,415 ).

In dieser Zeit ist also jedenfalls nur eine äusserst geringe Oxydationswärmebildung zu spüren. Dagegen schnellt die Wärmebildung bei Methylenblauzusatz und Sauerstoffdurchleiten herauf, um bei Abschluss sofort wieder abzusinken (das Plus gegenüber dem Sauerstoffabschluss ohne Methylenblau resultiert aus der nur allmählichen Erschöpfung des Sauerstoffvorrats).

Temperaturanstieg

f) $17,5 \mathrm{~g}$ Acetonhefe in $240 \mathrm{ccm} 1{ }^{0}{ }^{\prime} 0$ igem Fleischextrakt zeigt in $40^{\prime}$ ohne Sauerstoffdurchleiten $\mathbf{0 , 0 4 1 5 ^ { \circ }}$ $40^{\prime}$ mit Sauerstoffeinleiten . . . . . . $\mathbf{0 , 0 4 3}^{\circ}$ $40^{\prime}$ nachher ohne Sauerstoffeinleiten . . . $\mathbf{0 , 0 6 0 5}^{\circ}$ $2 \mathrm{~h} 20^{\prime}$ später ohne Sauerstoffeinleiten in $20^{\prime}$ $0,019^{\circ}=$. . . . . . . . . . . $0,038^{\circ}$ in 40 2 ccm Meth. dazu; $70^{\prime}$ mit Sauerstoffeinleiten $0,105^{\circ}$ oder. . . . . . . . . . . $\mathbf{0 , 0 6 0} 0^{\circ}$ in $40^{\prime}$ $60^{\prime}$ ohne Sauerstoffeinleiten $0,057^{\circ}$ oder. . $0,038^{\circ}$ in $40^{\prime}$

Hier ist jedenfalls sehon ohne Methylenblau ein Plus bei Sauerstoffgegenwart, in dem der höhere Wert uach Sauerstoffeinleiten noch auf den Verbrauch des gelösten Sauerstoffs gesetzt werden kann. Jedenfalls ist aber das berechnete Plus bei Sauerstoffeinleiten in Methylenblaugegenwart erbeblich höher als ohne dasselbe.

9. Wärmeversuche an Acetonhefe in neutraler und alkalischer Lösung.

Entsprechend den bei gleicher Reaktion gemachten Sauerstoffmessungen tritt auch eine messbare Oxydationswärme auf. Diese 
ist um so leichter messbar, als die Selbstgärung in diesen Fällen sehr rasch, oft so gut wie vollständig nachlässt (wegen Zerstörung der Invertase?).

a) $15 \mathrm{~g}$ Acetonhefe $1 / 2 \mathrm{~h}$ auf $100^{\circ}$ erhitzt, steril $230 \mathrm{ccm}$ Wasser. Suspension bis zur schwachen Rosafärbung gegen $\mathrm{Ph}$ mit $\mathrm{NaOH}$ versetzt. $1^{\mathrm{h}}$ ohne Sauerstoff hängen gelassen, darauf $50^{\prime}$ ohne Sauerstoff durchleiten . . . .0,001 ${ }^{\circ}$ $1 \mathrm{~h}$ mit Sauerstoffeinleiten 0,060 . . . . $0,050^{\circ}$ in $50^{\prime}$ Folgende $50^{\prime}$ ohne Sauerstoffeinleiten . . . 0,031 ${ }^{\circ}$

b) $18 \mathrm{~g}$ Acetonhefe in $240 \mathrm{ccm}$ Wasser (Gemisch wie oben alkalisch); steril; $1^{\mathrm{h}}$ hängen gelassen.

Darauf $30^{\prime}$ ohne Sauerstoffeinleiten . . . $\mathbf{0 , 0 0 3}^{\circ}$ $30^{\prime}$ mit Sauerstoffeinleiten . . . . . . $\mathbf{0 , 0 5 5}{ }^{0}$ 3 cem Methylenblau dazu; obne Sauerstoffeinleiten $12^{\prime} 0,001=$. . . . . $\mathbf{0 , 0 0 3}^{\circ}$ in $30^{\prime}$ mit Sauerstoff einleiten $12^{\prime} 0,052=\ldots . \mathbf{0 , 1 3 0}{ }^{\circ}$ in $30^{\circ}$

\section{III.}

Im vorstehenden ist gezeigt, dass durch Gegenwart von Methylenblau ein mit Wärmebildung verbundener Sauerstoffverbrauch stattfindet, der ohne dasselbe nicht bzw. in erheblich geringerem Grade vorhanden ist. .Nimmt man noch den von anderen Autoren gemachten Befund der Kohlensäurestimulierung von Methylenblau hinzu, so könnte man auf den Gedanken kommen, dass man Methylenblau als ein "atmungssteigerndes Mittel" betrachten müsse, wie solche von Warburg an. Seeigeleiern verschiedene $(\mathrm{NaCl}$, Metallspuren $\mathrm{NaOH}$ usw.) ${ }^{1}$ ) gefunden wurden. Indes lehrt eine nähere Betrachtung des Vorganges, dass eine solche Auffassung durchaus verfehlt wäre.

Die Aufhellung des Prozesses ist offenbar durch eine sehr interessante Untersuchung von Bredig und Sommer ${ }^{2}$ ) gegeben. Diese Autoren fanden folgendes: Versetzt man Ameisensäure oder Formaldehyd mit Methylenblau, so geschieht nichts, ebensowenig, wenn man kolloidales Platin zu einer Lösung von Methylenblau oder

1) Zeitschr. f. physiol. Chemie Bd. 66 S. 305. 1910.

2) Zeitschr. f. physik. Chemie Bd. 70 S. 34. 1909. 
Ameisensäure einzeln zusetzt. Versetzt man aber eine Lösung von Methylenblau und Ameisensäure (bzw. Formaldehyd) mit kolloidalem Platin, so wird das Methylenblau reduziert, während zugleich die Ameisensäure zu Kohlensäure oxydiert wird. Das Platin hat als Katalysator die Oxydation der Ameisensäure durch Methylenblau hervorgerufen bzw. „unendlich beschleunigt". Dabei bildet sich eine der reduzierten Methylenblaumenge sowie der verbrauchten Ameisensäure äquivalente Menge Kohlensäure.

Der Verlauf des Prozesses ist sehematisch ( $\mathrm{M}=$ Methylenblausalz) $\mathrm{M}+\mathrm{HCOOH} \rightarrow \mathrm{MH}_{2}+\mathrm{CO}_{2}$; es hat also eine Wasserstoffentziehung stattgefunden, die einer Oxydation gleich ist. Nun kann natürlich ebensognt eine Sauerstoffubertragung auf die organischen Moleküle wie eine Wasserstoffentziehung vor sich gehen; in diesem Fall würden die zur Reduktion von Methylenblau erforderlichen 2 H-Atome einer Spaltung des Wassermoleküls entstammen, das dabei seinen Sauerstoff einer Kohlensäureverbindung einverleibte ${ }^{1}$ ). - Durch Ermittelung der Reaktionskinetik wurde es vollends deutlich, dass das Platin als Katalysator dient und dabei die bekannten Aualogien mit organischen Enzymen aufweist. Ein solches methylenblaureduzierendes Enzym werden wir daher mit Wahrscheinlichkeit, dank zumal den von $\mathrm{Hahn}$ gefundenen zahlreichen Analogien zur Zymase, in rer Hefe annehmen müssen: eine "Reduktase", die man auch ebensogut als Oxydase bezeichnen könnte; denn, wie Bredig sagt: „Formal ist es natürlich gleichgültig, ob man die Reaktion als eine sauerstoffübertragende oder als eine in umgekehrter Richtung wasserstoffübertragende ansiebt, denn jede Oxydation eines Stoffes durch einen zweiten ist notwendig mit einer Reduktion dieses zweiten Stoffes verknüpft. Man kann daber die ,sauerstoffübertragende ${ }^{6}$ Wirkung des Platins vom Methylenblau auf den Formaldehyd oder auf die Ameisensäure sowohl als analog zur Wirkung einer ,Oxydase betrachten, wie auch als analog zur Wirkung einer Hydrogenase ${ }^{2}$ )."

Dieser durch Methylenblau hervorgerufene Oxydationsvorgang unterscheidet sich jedoch von dem vitalen gerade in demjenigen Moment, das nach Rubner's grundlegender Entdeckung das eigentlich

1) Dies ist z. B. bei der Oxydation von Formaldehyd zu Kohlensäure der Fall. Hier lautet die Gleichung: $\mathrm{HCOH}+2 \mathrm{M}+\mathrm{H}_{2} \mathrm{O} \rightarrow \mathrm{CO}_{2}+2 \mathrm{MH}_{2}$.

2) A. a. 0. S. 61 . 
Über schcinbare Atmung abgetöteter Zellen durch Farbstoffreduktion. 269

Wesentliche des Prozesses ist, in dem Energieumsatz. Nehmen wir beispielsweise die Oxydation von Methylalkohol zu Formaldehyd, einmal als vitalen Verbrennungsprozess, das andere Mal durch Reduktion von Methylenblau hervorgerufen.

Im ersten Fall haben wir:

$$
\underset{(63,4)}{\mathrm{CH}_{3} \mathrm{OH}}+\mathrm{O} \rightarrow \underset{(40,4)}{\mathrm{HCOH}}+\underset{(68,4) ;}{\left.\mathrm{H}_{2} \mathrm{O}^{1}\right)}
$$

pro Oxydation von $1 \mathrm{Mol}$. Methylalkohol werden also 45,4 Cal. frei. Jetzt verlaufe die Umwandlung von Methylalkohol zu Formaldehyd durch Methylenblaureduktion. Wir haben:

$$
\mathrm{CH}_{3} \mathrm{OH}+\mathrm{M} \rightarrow \mathrm{HCOH}+\mathrm{MH}_{2} \text {. }
$$

Man sieht, der Unterschied ist, dass hier anstatt Wasser Leukomethylenblau gebildet wird. Nun sind die Bildungswärmen von Methylenblau und seinen Umwandlungsprodukten nicht bekannt, auch nicht von ähnlich gebauten Körpern, soweit ich es in Erfahrung bringen konnte. Nur für die Reaktion Azobenzol $\rightarrow$ Hydrazobenzol berechnet sich aus den Bildungswärmen eine positive Wärmetönung von 25,7 Cal. pro Mol. ${ }^{1}$ ), also für eine gleiche Konstitutionsänderung. Da es jedoch zweifelhaft war, ob es gestattet ist, dies auf die Methylenblauhydrierung zu übertragen, führte ich selbst einige Messungen der Wärmetönung dieser Reaktion aus und erhielt im Mittel von drei Bestimmungen zufällig absolut genau denselben Wert, nämlich 25,7 Cal.

In Paranthese seien die Versuche geschildert. Benutzt wurde die Reduktion von Methylenblau in alkalischer Lösung mit Ammoniumsulfid. Diese verläuft bei überschüssigem Schwefelammon vollständig in wenigen Minuten nach der Gleichung: $\mathrm{NH}_{4} \mathrm{SH}+\mathrm{M}=\mathrm{NH}_{3}+\mathrm{S}+\mathrm{MH}_{2}$. Da die anderen hierbei auftretenden Reaktionswärmen bekannt sind, lässt sich durch die Differenz diejenige von $\mathrm{M}+\mathrm{H}_{2} \rightarrow \mathrm{MH}_{2}$ ermitteln. Die Reaktion lässt sich folgendermassen zerlegen:

1. $\mathrm{NH}_{4} \mathrm{SH}$ aq. $\rightarrow \mathrm{NH}_{3}$ aq. $+\mathrm{H}_{2} \mathrm{~S}$ aq. Diese Reaktion verläuft nach $\mathrm{T} k$ omsen mit einer negativen Wärmetönung von 6,2 Cal.

2. $\mathrm{H}_{2} \mathrm{~S} \rightarrow \mathrm{H}_{2}+\mathrm{S}$, nach $\mathrm{Th}$ omsen mit einer negativen Wärmetönung von 7,3 Cal. verbunden.

3. $\mathrm{H}_{2}+\mathrm{M} \rightarrow \mathrm{MH}_{2^{*}}$ Zu der gemessenen Wärmetönung der Gesamtreaktion müssen also + 13,5 Cal. addiert werden, um die gesuchte Reaktionswärme dieser Hydrierung zu finden.

$\mathrm{Zu}$ den Versuchen diente chemisch reines kristallisiertes Methylenblau, das mir durch das grosse Entgegenkommen der Höchster Farbwerke zur Verfügung

1) Bildungswärmen nach Landolt-Börnstein. 1905. 
stand ${ }^{1}$, and der Bernthsen' schen Formel entsprach: $\mathrm{C}_{16} \mathrm{H}_{18} \mathrm{~N}_{3} \mathrm{SUl}+3 \mathrm{H}_{2} \mathrm{O}$ also ein Molekulargewicht von 374,7 hatte. Pon einer genau 2\% igen Lösung dieses Salzes kamen in jedem Versuch $37,5 \mathrm{ccm}$ zur Verwendung, d. h. gerade 2. Millimol.

Die Versuche wurden in einer schon früher beschriebenen Anordnung vorgenommen ${ }^{2}$ ). Die $37,5 \mathrm{ccm}$ Methylenblaulösung wurden in einen Glaszylinder eingefïllt, der unten mit eingeschliffenem Stonfen versehen war, und in dem sich Rübrer und Beckmannthermometer befanden. . Diese Anordnung wurde in ein Dewergefäss gehängt, das mit durchschnittlich $220 \mathrm{ccm}$ alkalischer Ammonsulfidlösung gefüllt war (150 ccm 10 iger Ammonsulfid, $40 \mathrm{~cm} \frac{n}{10} \mathrm{NaOH}, 35 \mathrm{ccm}$ Wasser); das Dewergefäss wurde dann mit Propfen und einer Watte gefüllten Glasglocke, durch die nur Thermometer und Rührer hervorsah, verschlossen, in einem Wasserthermostaten von $26,5^{\circ}$ befestigt (die Innentemperatur wurde genan so gewählt) und 2-3 Stunden hängen gelassen, damit die Temperatur sich im Innern vollständig ausgleichen konnte. Wird jetzt durch Druck auf den Glasrührer von aussen der Glasstopfen des Zylinders herausgestossen, so fliesst das Methylenblau in die Ammoninmsulfidlösung, ohne dass der geringste Wärmeverlust stattfindet. Die Temperatur stieg sofort rapide, um dann allmählich wieder zu fallen und nach etwa 20-25 Minuten konstant zu werden. Ob der erste Anstieg nur durch verlangsamten Temperaturausgleich oder eine Zwischenreaktion bedingt ist, wurde nicht festgestellt, doch ist dies für das Resultat belanglos. Abgelesen wurde unmittelbar vor dem Durchstossen des Rährers nur eine halbe Stunde später. Diese Temperaturdifferenz entspricht der Wärmetönung der Gesamtreaktion. Die Thermostatenkorrektur betrug nur bis zu $0,002^{\circ}$. Würde in den Innenzylinder Wasser statt Methylenblaulösung gegeben, so fanden nur Temperaturveränderungen bis $z u \pm 0,010^{\circ}$ statt, welches also die Fehlerbreite der Methode angibt. Der Wasserwert des Gefässes wurde durch Abwiegen der Glasmasse und Vergleich mit dem gemessenen Wasserwert anderer Gefässe zu 28 bestimmt, die Wärmekorrektur der Flüssigkeiten gleich 1 gesetzt. Der Fehler spielt im Vergleich zu den Fehlern der Temperaturbestimmung keine Rolle. Im folgenden sind aus Temperaturanstieg und Gesamtwasserwert die erzeugten g-Oal. pro Versuchsmenge (2 Millimol. Methylenblau) berechnet und durch Addition von 13,5 Cal. zum halben Wert $(\times 1000)$ die Wärmetönung der Methylenblauhydrierung pro Mol. ausgerechnet.

\begin{tabular}{cccc}
$\begin{array}{c}\text { Temperatur- } \\
\text { anstieg }\end{array}$ & $\begin{array}{c}\text { Gesamt- } \\
\text { wasserwert }\end{array}$ & $\begin{array}{c}\text { g-Cal. in } \\
\text { Versuchs- } \\
\text { menge }\end{array}$ & $\begin{array}{c}\text { kg-Cal. der } \\
\text { Hydrierung } \\
\text { pro Mol. }\end{array}$ \\
1. $0,077^{0}$ & 286 & 22,0 & $\mathbf{2 4 , 5}$ \\
2. $0,387^{\circ}$ & 277 & 24,2 & $\mathbf{2 5 , 6}$ \\
3. $0,096^{\circ}$ & 280 & 26,9 & $\mathbf{2 6 , 9}$ \\
\cline { 3 - 4 } & & Durchschnitt & $\mathbf{2 5 , 7}$ Cal.
\end{tabular}

Setzen wir nun den so gewonnenen Wert von $+25,5$ Cal. für

1) Ich möchte auch hier dem Werke für die Überlassung dieses Präparates meinen besten Dank sagen.

2) Pflüger's Arch. Bd. 146 S. 165. 1912. 
Über scheinbare Atmung, abgetöteter Zellen durch Farbstoffreduktion. 271

die Reaktion $\mathrm{M}+\mathrm{H}_{2} \rightarrow \mathrm{MH}_{2}$ in die Gleichung der Methylalkoholoxydation ein, so können wir schreiben:

$$
\begin{gathered}
\mathrm{CH}_{3} \mathrm{OH}+\mathrm{M} \rightarrow \underset{(63,4)}{\mathrm{HCOH}}+\underset{(40,4) \quad}{\mathrm{MH}_{2}}(25,7) .
\end{gathered}
$$

Hierbei werden entgegen der obigen Reaktion des vitalen Verbrennungsvorganges pro Mol. Methylalkohol nur 2,7 Cal. frei, also nur etwa der 20. Teil. Nicht jede Reaktion ist gleich ungünstig; jedoch tritt stets gegenüber der vitalen Oxydation die Differenz von Wasserbildung und Methylenblauhydrierung auf, d. h. bei nach dem Schema verlaufenden Prozessen pro organischem Molekül ein Minus von 42,7 Cal. gegenüber der Verbrennungswärme ${ }^{\mathbf{1}}$ ).

Woher stammt nun die bei Methylenblaugegenwart gemessene Oxylationswärme? Daher, dass sich das in Wasser gelöste Leukomethylenblau durch den Sauerstoff der Luft wieder unter Wasserbildung oxydiert. Diese Reaktion $\mathrm{MH}_{2}+\mathrm{O} \rightarrow \mathrm{M}+\mathrm{H}_{2} \mathrm{O}$ verläuft mit der im obigen Fall ausgebliebenen Wärmetönung von 42,7 Cal. pro Molekül, und damit ist der Ring geschlossen. Diese zweite nachträgliche Oxydation des Methylenblaus hat aber mit der Reduktionsfähigkeit der Zelle gar nichts zu scbaffen. Sieverläuft ausserhalb, in der Lösung, mit oder ohne Gegenwart von Zellen oder Zellstoffen, und diese Wärme, d. h. der weitaus grösste Teil der frei werdenden Energie des Prozesses, ist der Zelle verloren gegangen.

Anders dagegen, wenn, wie Bredig andeutet, sein System: Formaldehyd - Methylenblau - kolloidales Platin, insofern ein Modell des vitalen Vorgangs darstellt, dass ebensowohl als für Platin ein Enzym auch für Methylenblau als Sauerstoffüberträger ein entsprechender Körper in der lebenden Zelle als Analogon angenommen würde. Ein solcher in der Zelle befindlicher und aus ihr nicht diffusibler Körper könnte bei seiner Wiederoxydation der Zelle die freiwerdende Energie zugute kommen lassen. Eine solche Konstruktion hat sogar eine gewisse Wahrscheinlichkeit für sich; und es erscheint nicht ausgeschlossen, dass der methylenblauredigierende Enzym auch im normalen Atmungsvorgang eine Rolle spielt. In

1) Für die Umwandlung: Formaldehyd $\mathrm{zu} \mathrm{CO}_{2}$ berechnet sich als Verbrennungsvorgang pro Mol. 23 Cal., bei Redultion von Methylenblau $37 \mathrm{Cal}$. Pflüger's Archiv für Physiologie. Bd, 149. 
dieser Richtung kann das von Ehrlich inaugurierte Studium der vitalen Farbstoffreduktion sicherlich noch wichtige Erkenntnisse zutage fördern.

\section{IV.}

Auf Grund seiner Farbstudien hat Ehrlich seine Lehre der "Sauerstoffnot der Zelle" der P fl ü ge r'schen vom Sauerstoffüberfluss entgegengestellt. Die auch heute noch zwischen verschiedenen Forschern berrschende Meinungsverschiedenheit über den Sauerstoffgehalt der Zellen hat teilweise jederfalls darin ihren Grund, dass die Bedingungen für die verschiedenen Zellen und Zellarten nicht einheitlich sind. Nach Pfeffer und Pflüger regelt die lebendige Zelle die Grösse des Sauerstoffverbrauchs selbst ${ }^{1}$ )", was natürlich nur bei Überschuss von verfügbarem Sauerstoff möglich ist: "Die tierische Verbrennung", sagt Pflüger, "setzt nicht bloss keinen aktiven und nur neutralen Sauerstoff voraus, sondern ist auch innerhalb weiter Grenzen vollkommen unabhängig vom Partialdruck des neutralen Sauerstoffs ${ }^{2}$ )."

$\mathrm{Sch}$ ematisch lässt sich dies nicht auf alle Organismen übertragen. Wie $\mathrm{Hen}_{\mathrm{z}}{ }^{3}$ ) gezeigt hat, können bei ungünstiger Sauerstoffversorgung in vielzelligen Organismen die aussengelegenen Zellen schon einen so grossen Teil des hinzudiffundierenden Sauerstoffs abfangen, dass die tiefergelegenen normaliter mangelhaft mit Sauerstoff versorgt sind und dafür vielleicht anaeroben Umsatz haben, der bei vermehrter Sauerstoffzufuhr in aeroben umschlägt. Bei höheren Organismen wird eine weitere Komplikation dadurch gegeben, dass für eine stärkere Dissoziation des Hämoglobins ein sehr niedriger Partialdruck des Sauerstoffs in den Geweben Voraussetzung ist. Tatsächlich findet hier Ver zà $\mathbf{r}^{4}$ ) im Muskel Proportionalität $z$ wischen äusserem Sauerstoffdruck und Gaswechsel, also keinen messbaren Partialdruck im Muskel, während der Sauerstoffverbrauch der Speicheldrüsen vom Partialdruck unabbängig ist und der der Nieren sogar mit sinkendem Partialdruck steigt. Beweist schon dieser letztere Fall

1) Pflüger, Pflüger's Arch. Bd. 10 S. 251, ebenso Bd. 6 S. 43; vgl. auclı Pfeffer, Pflanzenphysiologie, 2. Aufl., Bd. 1 S. 547.

2) Pflüger, a. a. 0.

3) Biochem. Zeitschr. Bd. 26 S. 255. 1910. Das gleiche lässt sich mit aufeinandergeschichteten Seeigeleiern nachahmen, während deren Atmung, wie Warburg und Henze gezeigt haben, bei genügender Verteilung im Seewasser vom Partialdruck des Sauerstoffes unabhängig ist.

4) Journ. of Physiol. vol, 45 p. 39. 1912. 
eine indirekte Wirkung des Sauerstoffdruckes, so ist an eine solche auch bei anderen tätigen Zellen zu denken, z. B. bei sich bewegenden oder wachsenden ${ }^{1}$ ). Demgegenüber kann wohl auch jetzt noch für die einzelne ruhende Zelle mit grosser Allgemeinheit der Satz vertreten werden, dass ihre Verbrennungen vom Partialdruck unabhängig sind, und sie demgemäss auch einen Sauerstoffüberschuss enthalten muss. Nun hat man gleichwohl z. B. die Befunde W a r b u rg's, dass durch die Membranbildung der Seeigeleier die Atmungsgrösse aufs sechsfache gesteigert wird, mit Durchlässigkeitsänderungen der Oberfläche für Sauerstoff zusammen gebracht. Ich möchte deshalb durch folgenden Versuch, den ich vor einem Jahre in Neapel ausgeführt habe, demonstrieren, dass im Seeigelei gelöster Sauerstoff vorhanden ist, und dass sein Eindringen gleich schnell geschieht in befruchtete Kier, unbefruchtete und solche Eier, deren Atmung durch $1 / 500 \mathrm{n}$. Phenylurethan etwa auf die Hälfte herabgesetzt ist.

Hierzu diente ein von $\mathrm{Unna}^{2}$ ) angegebenes Gemisch von Leukomethylenblau mit einem starken Reduktionsmittel, das, als Rongalit bezeichnet, eine Verbindung von Formaldehyd mit Sulfoxylsäure ist. Dieses zweifellos giftige Gemisch („Rongalitweiss“) färbt in schon so minimaler Konzentration die Seeigeleier, dass dieselben sich in mit einem Tropfen Reagens versetztem Seewasser ungeschädigt zu tiefblau gefärbten schwimmenden Larven entwickeln können.

Setzt man einen Tropfen Rongalitweiss zu sauerstofffreiem Wasser, so bleibt es farblos; dringt aber Sanerstoff ein, so färbt es sich blau gemäss dem Eindringen des Sauerstoffs. Ebenso blau färben sich die Eier; doch könnte man das noch auf eine Hineindiffusion des im Wasser gebildeten Methylenblaus statt des Sauerstoffs bezieben. Es wurde deshalb folgender Versuch gemacht: Seeigeleier wurden in einen Tropftrichter eingefüllt, der unten mit Watte ausgelegt war, und durch die Eiersuspension sowie eine anschliessende grössere Flasche mit Seewasser $3 / 4$ Stunde lang Wasserstoff durchgeleitet, um allen Sauerstoff zu entfernen. Jetzt wurde aus einer schon vorher in dem Hals des Trichters befestigten Pipette ein Tropfen Rongalitweiss zu der Eiersuspension gesetzt, die farblos

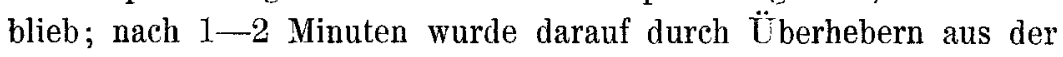

1) Andeutungen davon ergaben sich bei Vibrio-Metschnikoff, Doch ist dies noch nicht näher untersucht worden. Vgl. O. Meyerh of, Sitzungsber. Heidelb. Akad. d. Wissensch., naturw. Klasse B. 1912 S. 1.

2) Arch. f. mikr. Anat. Bd. 78 S. 1. 1911. 
grossen Flasche mit sauerstofffreiem Seewasser das Rongalitweiss weggespült, wobei das Wasser aus dem Tropftrichterrobr abfloss, der grössere Teil der Eier aber durch die Watte festgehalten wurde.

Schliesslich wurde die Watte mit den Eiern herausgenommen und in ein Uhrschälehen mit sauerstoffhaltigem Seewasser ausgeschüttelt, das sofort unter dem Mikroskop betrachtet wurde. Die anfangs ganz farblosen Eier färbten sich jetzt in farbfreiem Seewasser allmählich, und zwar gleich schnell diejenigen, die in ein Schälchen mit Phenylurethan-Seewasser gekommen waren wie die anderen, die teils befruchtet, teils unbefruchtet waren. Die Färbung machte bei einer mässig starken Bläuung halt (weil jedenfalls sehr stark weggewaschen war). Doch nahm diese Bläuung bei Abtötung eines Teils mit Blausäure nicht im geringsten zu. Am nächsten Morgen war ein Teil der Eier zerstört, doch fandeu sich zahlreiche normal aussehende blaue schwimmende Larven in der Flüssigkeit. Dieser Versuch lässt keine andere Deutung zu, als dass der Sanerstoff leicht in die lebende Zelle eindringt und in ihr in gelöstèr Form vorhanden ist.

\section{Zusammenfassung.}

1. Es wird gezeigt, dass neutrale und schwach alkalische Acetonhefe eine gut messbare selbständige Sauerstoffzehrung hat, dass sie aber bei Gegenwart von Methylenblau das Mehrfache an Sauerstoff der Luft verbraucht wie ohne dasselbe. Ein derartiger Unterschied lässt. sich auch aus Wärmemessungen in saurer und alkalischer Lösungentnehmen.

2. Es wird aus den angeführten Versuchen, Messungen der Reduktionsgeschwindigkeit, sowie aus thermochemischen Überlegungen (in Verbindung mit einer Bestimmung der Wärmetönung der Methylenblauhydrierung) gefolgert, dass die Methylenblaureduktion der getöteten Hefezelle etwas von dem natürlichen Verbrennungsprozess quantitativ Versehiedenes und Unabhängiges ist. Der Mechanismus. derselben wird auf Grund einer Bredig'schen Arbeit erklärt.

3. Es wird nach einer Diskussion der Pflüger'schen Lehre von dem Sauerstoffüberfluss der Zelle durch ein Experiment demonstriert, dass in einer lebenden tierischen Zelle, dem Seeigelei, gelöster Sauerstoff vorhanden ist. 